\title{
A NOTE ON A THEOREM OF GEHRING AND LEHTO
}

\author{
A. G. FADE LL
}

ABSTRACT. The concept of mesh approximate differential is defined as a modification of regular approximate differential. It is shown that for open continuous real-valued maps on open sets in $n$-space the concepts of mesh approximate differentiability and total differentiability are equivalent, and the Gehring-Lehto theorem is obtained as a corollary by a sharpening of a known theorem on regular approximate differentials.

1. Introduction. A theorem of Gehring and Lehto [5], [9] asserts the existence almost everywhere (a.e.) of a total differential for an open continuous map $f: S \rightarrow R^{n}$ on an open set $S$ in Euclidean $n$-space $R^{n}$, assuming that $f$ has a total differential a.e. on $S$ with respect to $n-1$ variables. The present note obtains this theorem as a corollary of the equivalence of mesh approximate differentials (Definition 3) and total differentials in the class of real-valued open continuous maps on open sets in $R^{n}$ (Theorem 1 ), by applying a theorem of the author [3]. Thus any available sufficient conditions for the existence a.e. of a mesh approximate differential will yield total differentiability a.e. within the class of open continuous maps on open sets.

2. Notation, definitions, and basic lemmas. Let $x=\left(x^{1}, \cdots, x^{n}\right)$ denote points in Euclidean $n$-space $R^{n},|x-y|=\left[\sum_{i=1}^{n}\left(x^{i}-y^{i}\right)^{2}\right]^{1 / 2}$ the distance between $x$ and $y$, and $x \cdot y=x^{1} y^{1}+\cdots+x^{n} y^{n}$ the usual inner product.

Definition 1. For $x_{0} \in R^{n}$ let $\mathcal{H}\left(x_{0}\right)$ denote a family of oriented $(n-1)$ hyperplanes (parallel to the coordinate planes) such that $x_{0}$ is a point of linear density of $\bigcup_{H, H} \in \mathcal{H}\left(x_{0}\right)$, in the direction of each coordinate axis. Then we term $\mathcal{H}\left(x_{0}\right)$ a thick regular mesh of $(n-1)$-hyperplanes at $x_{0}$.

Definition 2. For $x_{0} \in R^{n}$ let $\mathcal{C}\left(x_{0}\right)$ denote a family of oriented $n$-cubes (faces parallel to the coordinate planes) centered at $x_{0}$ such that $x_{0}$ is a point of density of $\bigcup$ fr $C, C \in \mathcal{C}\left(x_{0}\right)$. Then we term $\mathcal{C}\left(x_{0}\right)$ a thick regular family of $n$-cubes at $x_{0}$.

Received by the editors February 27, 1974.

AMS (MOS) subject classifications (1970). Primary 26A54.

Key words and phrases. Total differential, regular approximate differential, mesh approximate differential, open continuous mapping. 
Note that given $\mathcal{H}\left(x_{0}\right)$ there exists $\mathcal{C}\left(x_{0}\right)$ such that $\bigcup_{\text {fr }}, C \in \mathcal{C}\left(x_{0}\right)$, is a subset of $\bigcup H, H \in \mathcal{H}\left(x_{0}\right)$. To see this observe first that $x_{0}$ is a point of linear density of $\bigcup_{H}, H \in \mathcal{H}\left(x_{0}\right)$, in the direction of each coordinate axis, and then consider for each $i=1, \cdots, n$ the intersections $A_{i}, B_{i}$ of this union of hyperplanes with the two rays from $x_{0}$ parallel to the $i$ th coordinate axis. Finally construct the family $\mathcal{C}\left(x_{0}\right)$ by taking faces through points of $A_{i}^{\prime}, B_{i}^{\prime}$ $(i=1, \cdots, n)$, where $A_{i}^{\prime}$ and $B_{i}^{\prime}$ are those subsets of $A_{i}$ and $B_{i}$, respectively, which are congruent to the common intersection of say $A_{1}$ with suitable rotations of all the $A_{i}$ and $B_{i}(i=1, \ldots, n)$ about $x_{0}$.

Definition 3. Let $f: S \rightarrow R$ be a real-valued function on an open set $S$ of $R^{n}$, and let $A$ be a subset of $S$. Assume that $f$ has partial derivatives $f_{i}^{\prime}\left(x_{0}\right), i=1, \cdots, n$, at $x_{0} \in A$ relative to $A$, and let

$$
e_{A}\left(x, x_{0}\right)=\frac{\left|f(x)-f\left(x_{0}\right)-\nabla f\left(x_{0}\right) \cdot\left(x-x_{0}\right)\right|}{\left|x-x_{0}\right|}, \quad x \in A, x \neq x_{0},
$$

where $\nabla f\left(x_{0}\right)=\left(f_{1}^{\prime}\left(x_{0}\right), \cdots, f_{n}^{\prime}\left(x_{0}\right)\right)$. If $e_{A}\left(x, x_{0}\right) \rightarrow 0$ as $x \rightarrow x_{0}$ we say that $f$ has a total differential at $x_{0}$ relative to $A$. Then the expressions total differential at $x_{0}$, regular approximate differential at $x_{0}$, and mesh approximate differential at $x_{0}$ apply to the cases $A=S, A=\bigcup(\operatorname{fr} C) \cap S$,

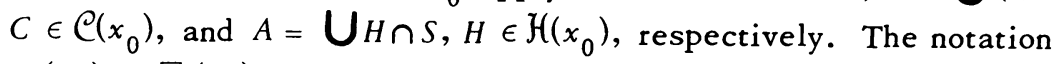
$D f\left(x_{0}\right) h=\nabla f\left(x_{0}\right) \cdot h$ will apply to each of these differentials and the context of the usage will avoid any confusion.

Lemma 1. Let $x_{0}$ be a point of (linear) density of a measurable set $A$ $C R$. Then for every $\epsilon>0$ there exists $\delta>0$ such that if $\left|x-x_{0}\right|<\delta, x \epsilon$ $R$, there correspond points $a, b \in A$ such that $a<x<b,|x-a|<\epsilon\left|x-x_{0}\right|$, and $|x-b|<\epsilon\left|x-x_{0}\right|$.

Proof. This is a direct consequence of the definition of point of linear density. The details are found essentially in [5, p. 7] and [9, p. 10].

Lemma 2. Let $\mathcal{H}\left(x_{0}\right)$ be a thick regular mesh of $(n-1)$-hyperplanes at $x_{0} \in S$ where $S$ is open set in $R^{n}$. Then for every $\epsilon>0$ there exists $\delta>0$ such that if $\left|x-x_{0}\right|<\delta$ there corresponds to $x$ an $n$-interval $I_{x}=\left\{y: a^{i} \leq\right.$

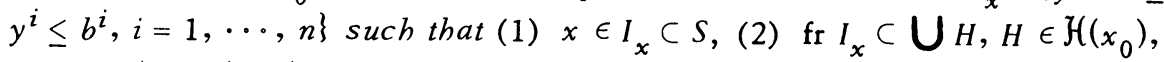
and (3) $|y-x|<\epsilon\left|x-x_{0}\right|$ for every $y \in \operatorname{fr} I_{x}$.

Proof. Via Lemma 1 , since $x_{0}$ is a point of linear density of $\bigcup_{H}, H \in \mathcal{H}\left(x_{0}\right)$, in the direction of each coordinate axis, for a given 
$\epsilon>0$ there exist $\delta_{i}>0$ such that if $\left|x^{i}-x_{0}^{i}\right|<\delta_{i}$ there correspond $H_{1}^{i}, H_{2}^{i} \in \mathcal{H}\left(x_{0}\right)$ such that $H_{1}^{i}, H_{2}^{i}$ intersects the $i$ th coordinate axis in the points $a_{i}, b_{i}$ satisfying $a^{i}<x^{i}<b^{i},\left|x^{i}-a^{i}\right|<\epsilon\left|x^{i}-x_{0}^{i}\right|$, and $\left|x^{i}-b^{i}\right|<$ $\epsilon\left|x^{i}-x_{0}^{i}\right|, i=1, \cdots, n$. Finally, choosing $\delta_{0}$ so that

$$
\left\{x:\left|x-x_{0}\right|<\delta_{0}\right\} \subset S \text {, }
$$

set $\delta=\min \left(\delta_{0}, \delta_{1}, \cdots, \delta_{n}\right)$ and $I_{x}=\left\{y: a^{i} \leq y^{i} \leq b^{i}, i=1, \cdots, n\right\}$.

\section{Main theorems.}

Theorem 1. Let $f: S \rightarrow R$ be a real-valued open continuous mapping on an open set $S$ in $R^{n}$. If $f$ has a mesh approximate differential at $x_{0} \in S$, then $f$ has a total differential at $x_{0}$.

Proof. By hypothesis there exists a mesh $\mathcal{H}\left(x_{0}\right)$ of oriented $(n-1)$ hyperplanes such that $x_{0}$ is a point of density of $A=\bigcup_{H \cap S}, H \in \mathcal{H}\left(x_{0}\right)$, and the total differential $D f\left(x_{0}\right)$ exists at $x_{0}$ relative to $A$. Let $\epsilon>0$ be given and choose $\delta_{1}>0$ such that the conclusion of Lemma 2 is satisfied for $\delta_{1}=\delta$. Suppose $\left|x-x_{0}\right|<\delta_{1}, x \in S$, and $I_{x}$ is the $n$-interval available from Lemma 2. Since $f$ is an open continuous map the function $h: y \rightarrow f(y)-$ $f\left(x_{0}\right)-D f\left(x_{0}\right)\left(x-x_{0}\right)$ restricted to $y \in I_{x}$ assumes its maximum on the frontier of $I_{x}$, say $h\left(y^{*}\right), y^{*} \in$ fr $I_{x}$, and $\left|y^{*}-x\right|<\epsilon\left|x-x_{0}\right|$. Observe that if we assume, as we now do, that $\epsilon<1$, then $\left|y^{*}-x_{0}\right|<2\left|x-x_{0}\right|$. Accordingly, we have

$$
\begin{aligned}
\left|f(x)-f\left(x_{0}\right)-D f\left(x_{0}\right)\left(x-x_{0}\right)\right| \leq\left|f\left(y^{*}\right)-f\left(x_{0}\right)-D f\left(x_{0}\right)\left(x-x_{0}\right)\right| \\
\leq\left|f\left(y^{*}\right)-f\left(x_{0}\right)-D f\left(x_{0}\right)\left(y^{*}-x_{0}\right)\right|+\left|D f\left(x_{0}\right)\left(y^{*}-x\right)\right| \\
<\epsilon\left|y^{*}-x_{0}\right|+\left|\nabla f\left(x_{0}\right)\right|\left|y^{*}-x\right|<\left(2+\left|\nabla f\left(x_{0}\right)\right|\right) \epsilon\left|x-x_{0}\right|
\end{aligned}
$$

if $\left|x-x_{0}\right|<\min \left(\delta_{1}, \delta_{2}\right)$ where $\delta_{2}$ is selected so that we have

$$
\left|f(z)-f\left(x_{0}\right)-D f\left(x_{0}\right)\left(z-x_{0}\right)\right|<\epsilon\left|z-x_{0}\right|
$$

if $z \in A$ and $\left|z-x_{0}\right|<\delta_{2}$. Thus $f$ has a total differential at $x_{0}$ and the proof is complete.

A theorem of the author [3] asserts that for continuous real-valued maps on open sets in $R^{n}$ the existence a.e. of a regular approximate differential (Definition 3 ) is a consequence of the existence a.e. of a total differential with respect to $n-1$ variables. Inspection of the proof reveals that "regular approximate" can be strengthened to "mesh approximate" by considering instead of a face of an $n$-cube the $(n-1)$-hyperplane containing the face. For reference we state this result formally. 
Theorem 2. Given a continuous real-valued map $f: S \rightarrow R$ on an open set $S$ in $R^{n}$, suppose $f$ has a total differential a.e. with respect to $n-1$ variables. Then $f$ has a mesh approximate differential a.e. on $S$.

In view of Theorem 1 we infer from Theorem 2 the theorem of Gehring and Lehto for real-valued maps, which when applied to the coordinate maps of a given map $f: S \rightarrow R^{m}$ yields the following form of the theorem:

Gehring-Lehto theorem. Given a map $f: S \rightarrow R^{m}$ on an open set $S$ in $R^{n}$, suppose the $m$ coordinate maps are continuous open maps on $S$. Then if $f$ has a total differential a.e. on $S$ with respect to $n-1$ variables, then $f$ has a total differential a.e. on $S$.

An example of Väisälä [9, p. 11], shows that in the Gehring-Lehto theorem for $n \geq 3$ the hypothesis of total differentiability with respect to $n-1$ variables a.e. may not be replaced by the existence a.e. of simply partial derivatives. Accordingly since the theorem is a consequence of Theorem 1 and Theorem 2, we remark that the existence a.e. of partial derivatives for $n \geq 3$ does not imply the existence a.e. of a mesh approximate differential a.e., so that in a certain sense Theorem 2 is sharp.

\section{REFERENCES}

1. A. P. Calderón, On the differentiability of absolutely continuous functions, Riv. Mat. Univ. Parma 2 (1951), 203-213. MR 13, 544.

2. L. Cesari, Sulle funzioni assolutamente continue in due variabili, Ann. Scuola Norm. Sup. Pisa (2) 10 (1941), 91-101. MR 3, 230.

3. A. G. Fadell, On the existence of regular approximate differentials, Proc. Amer. Math. Soc. 37 (1973), 541-544. MR 47 \#2016.

4. - A generalization of Rademacher's theorem on complete differential, Colloq. Math. 27 (1973), 125-131.

5. F. W. Gehring and O. Lehto, On the total differentiability of functions of a complex variable, Ann. Acad. Sci. Fenn. Ser. A I No. 272 (1959), 9 pp. MR 23 \#A1799.

6. O. Lehto and K. I. Virtanen, Quasikonforme Abbildungen, Die Grundlehren der math. Wissenschaften in Einzeldarstellungen mit besonderer Berücksichtigung der Anwendungsgebiete, Band 126, Springer-Verlag, Berlin and New York, 1965. MR 32 \#5872.

7. H. M. Riemann, Ueber das Verhalten von Flachen unter quasiconformen Abbildungen im Raum, Ann. Acad. Sci. Fenn. Ser. A I No. 470 (1970), 1-26.

8. J. Serrin, On the differentiability of functions of several variables, Arch. Rational Mech. Anal. 7 (1961), 359-372. MR 25 \#3131.

9. J. Väisälä, Two new characterizations for conformality, Ann. Acad. Sci. Fenn. Ser. A I No. 362 (1965), 12 pp. MR $30 \# 4975$.

DEPARTMENT OF MATHEMATICS, STATE UNIVERSITY OF NEW YORK AT BUFFALO, AMHERST, NEW YORK 14226 